\title{
Sclerosing Perivascular Epithelioid Cell Tumor of the Lung: A Case Report with Cytologic Findings
}

\author{
Ha Yeon Kim · Jin Hyuk Choi \\ Hye Seung Lee · Yoo Jin Choi \\ Aeree Kim · Han Kyeom Kim \\ Department of Pathology, Korea University Guro \\ Hospital, Seoul, Korea \\ Received: January 21, 2016 \\ Revised: February 11, 2016 \\ Accepted: February 19, 2016

\section{Corresponding Author} \\ Han Kyeom Kim, MD, PhD \\ Department of Pathology, Korea University Guro \\ Hospital, 148 Gurodong-ro, Guro-gu, Seoul 08308 \\ Korea \\ Tel: +82-2-2626-3251 \\ Fax: $+82-2-2626-1481$ \\ E-mail: sswords@naver.com
}

\begin{abstract}
Benign perivascular epithelioid cell tumor (PEComa) of the lung is a rare benign neoplasm, a sclerosing variant of which is even rarer. We present a case of 51-year-old man who was diagnosed with benign sclerosing PEComa by percutaneous fine needle aspiration cytology and biopsy. The aspirate revealed a few cell clusters composed of bland-looking polygonal or spindle cells with fine granular or clear cytoplasm. Occasional fine vessel-like structures with surrounding hyalinized materials were seen. The patient later underwent wedge resection of the lung. The histopathological study of the resected specimen revealed sheets of polygonal cells with clear vacuolated cytoplasm, variably sized thin blood vessels, and densely hyalinized stroma. In immunohistochemical studies, reactivity of tumor cells for human melanoma black 45 and Melan-A further supported the diagnosis of benign sclerosing PEComa. To the best of our knowledge, this is the first case of benign sclerosing PEComa described in lung.
\end{abstract}

Key Words: Lung neoplasms; Solitary pulmonary nodule; Perivascular epithelioid cell neoplasms
Benign perivascular epithelioid cell tumor (PEComa) of the lung is a rare benign neoplasm. It is also called benign clear cell "sugar" tumor due to its glycogen content in cytoplasm. Since initially described by Liebow and Castleman in 1963, ${ }^{1}$ about 50 cases have been reported so far in the English literature. Histologically, benign PEComa is composed of sheets of epithelioid or spindle cells with clear cytoplasm and thin walled vascular spaces. Cytological findings of benign PEComa include loosely cohesive clusters of polygonal or spindle cells with fine granular or clear cytoplasm. To our knowledge, the cytologic findings of benign PEComa of the lung have been described in only three reports to date, and benign sclerosing PEComa was not mentioned in those reports. ${ }^{2-4}$ In 2008, a distinctive variant of benign PEComa with $>50 \%$ stromal hyalinization was designated as benign "sclerosing" PEComa by Hornick and Fletcher. ${ }^{5}$ We present cytologic findings of a benign sclerosing PEComa of the lung.

\section{CASE REPORT}

We report a case of a 51-year-old male patient diagnosed with benign PEComa by percutaneous fine needle aspiration of the lung. He had hypertension, diabetes mellitus and history of smoking (30 pack-years). He complained no specific symptoms. On chest X-ray, a well-demarcated mass was incidentally found in the periphery of the right upper lobe. It measured $1 \mathrm{~cm}$ and was confined to lung parenchyma.

Computed tomography-guided percutaneous aspiration and gun biopsy was simultaneously performed. The lesion was diagnosed as benign sclerosing PEComa on the basis of cytologic and histologic findings. After the diagnosis, the patient underwent subsequent wedge resection of the lung. The histologic and immunohistochemical findings of the resected specimen confirmed the diagnosis of benign sclerosing PEComa.

\section{Cytologic findings}

Alcohol-fixed liquid-based preparation (ThinPrep, Cytyc Corporation, Boxborough, MA, USA) was performed after percutaneous fine needle aspiration. The cytologic preparation was hypocellular with a few cell clusters in a clean background. These clusters consisted of polygonal to spindle cells with oval nuclei and small distinct nucleoli. Most of the cells showed abundant basophilic and granular cytoplasm or clear intracytoplasmic vac- 
uoles (Fig. 1A). Nuclear pleomorphism was minimal. Neither necrosis nor mitotic figures was observed. Some of the clusters had thin vessel-like structures within (Fig. 1B). Semitranslucent hyalinizing material was noted around the vascular structures (Fig. 1C).

\section{Histologic findings of biopsied specimen}

Gun-biopsied specimen was also composed of bland-looking polygonal cells with vacuolated cytoplasm and minimal atypia. Variable-sized vascular spaces were observed among sheets of tumor cells. Dilated vessels were surrounded by hyalinized stroma. Focal microcalcification was noted. Tumor cells were positive for human melanoma black 45 (HMB-45). Intracytoplasmic vacuoles were confirmed as glycogen by periodic acid-Schiff stain and diastase periodic acid-Schiff stain.

\section{Gross findings of resected specimen}

Wedge resected specimen of the right upper lobe revealed a 1.0-cm-sized, well-demarcated, non-encapsulated, and round mass. It was well confined within the pulmonary parenchyma. The mass was tan-colored and had a firm texture. The cut surface of the mass showed multiple tiny cyst-like spaces at the periphery.

\section{Histologic findings of resected specimen}

On light microscopy, the histologic findings of the surgical specimen were similar to those of the gun-biopsied specimen. The tumor was mainly composed of sheets of polygonal cells with distinct cell borders. Tumor cells showed clear cytoplasmic vacuoles and round to oval nuclei with granular chromatin and distinct nucleoli (Fig. 2A). Numerous dilated, thin walled blood vessels were present throughout the tumor. Those vessels were surrounded by pinkish hyalinized stroma (Fig. 2B). A few microcalcifications were seen. The cytoplasmic vacuoles were revealed to be glycogen on periodic acid-Schiff stain and diastase periodic acid-Schiff stain (Fig. 3A, B). Those vacuoles were not
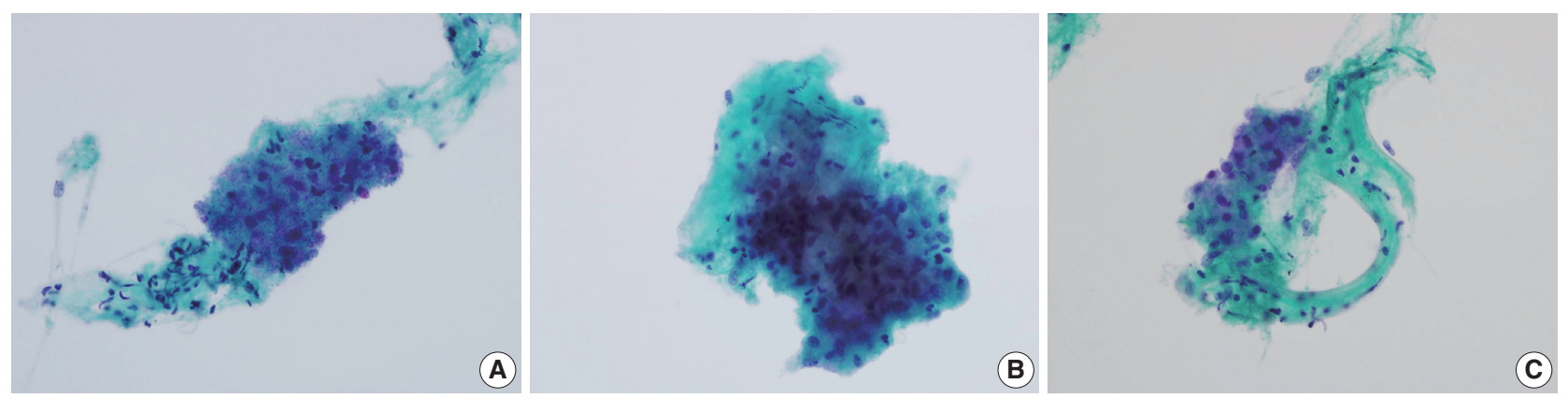

Fig. 1. Liquid-based aspiration cytology of benign sclerosing perivascular epithelioid cell tumor. (A) A few cohesive clusters of polygonal to spindle-shaped bland cells in a clean background (Papanicolaou staining). (B) The tumor cells show oval nuclei, small distinct nucleoli and abundant basophilic and granular cytoplasm. Most cells contain clear intracytoplasmic vacuoles (Papanicolaou staining). (C) Thin-walled blood vessels are occasionally seen. Semitranslucent hyalinizing material was noted around the vascular structure (Papanicolaou staining).
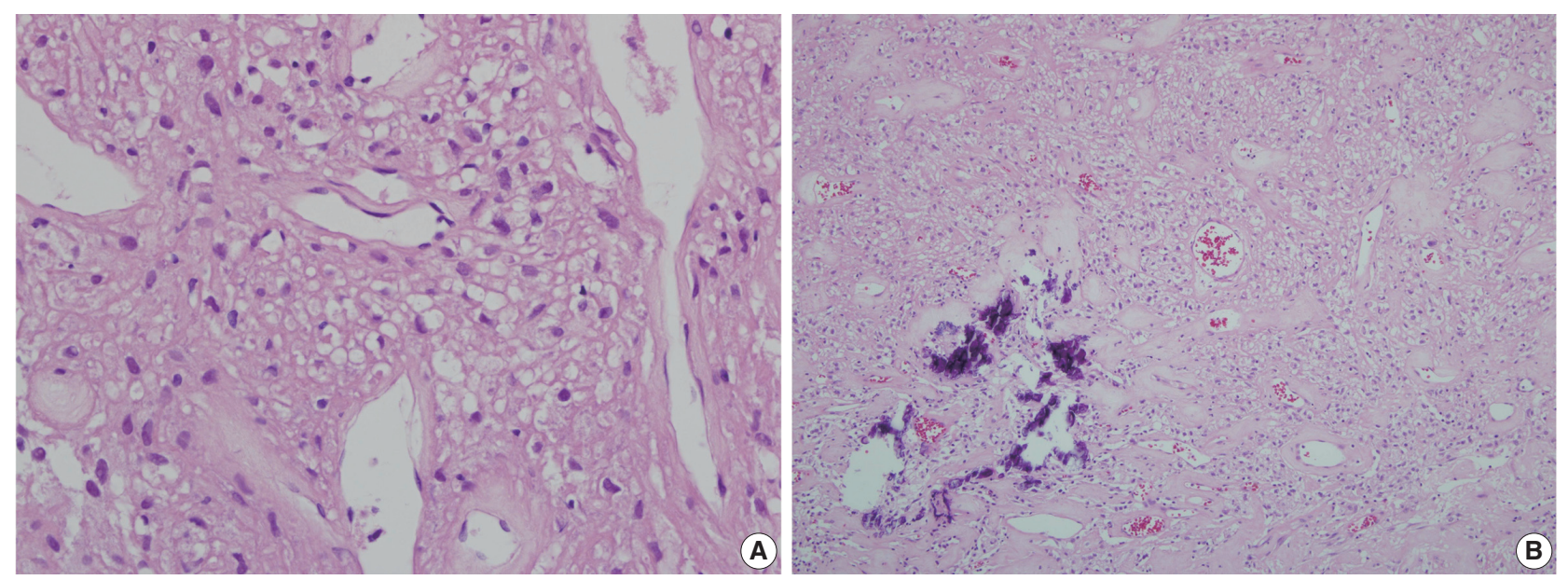

Fig. 2. Histologic section of benign sclerosing perivascular epithelioid tumor. (A) Dilated vessels surrounded by collagenous stroma and polygonal cells with clear cytoplasm. (B) Sheet-like arrangement of tumor cells around various-sized blood vessels and a focus of microcalcification. 

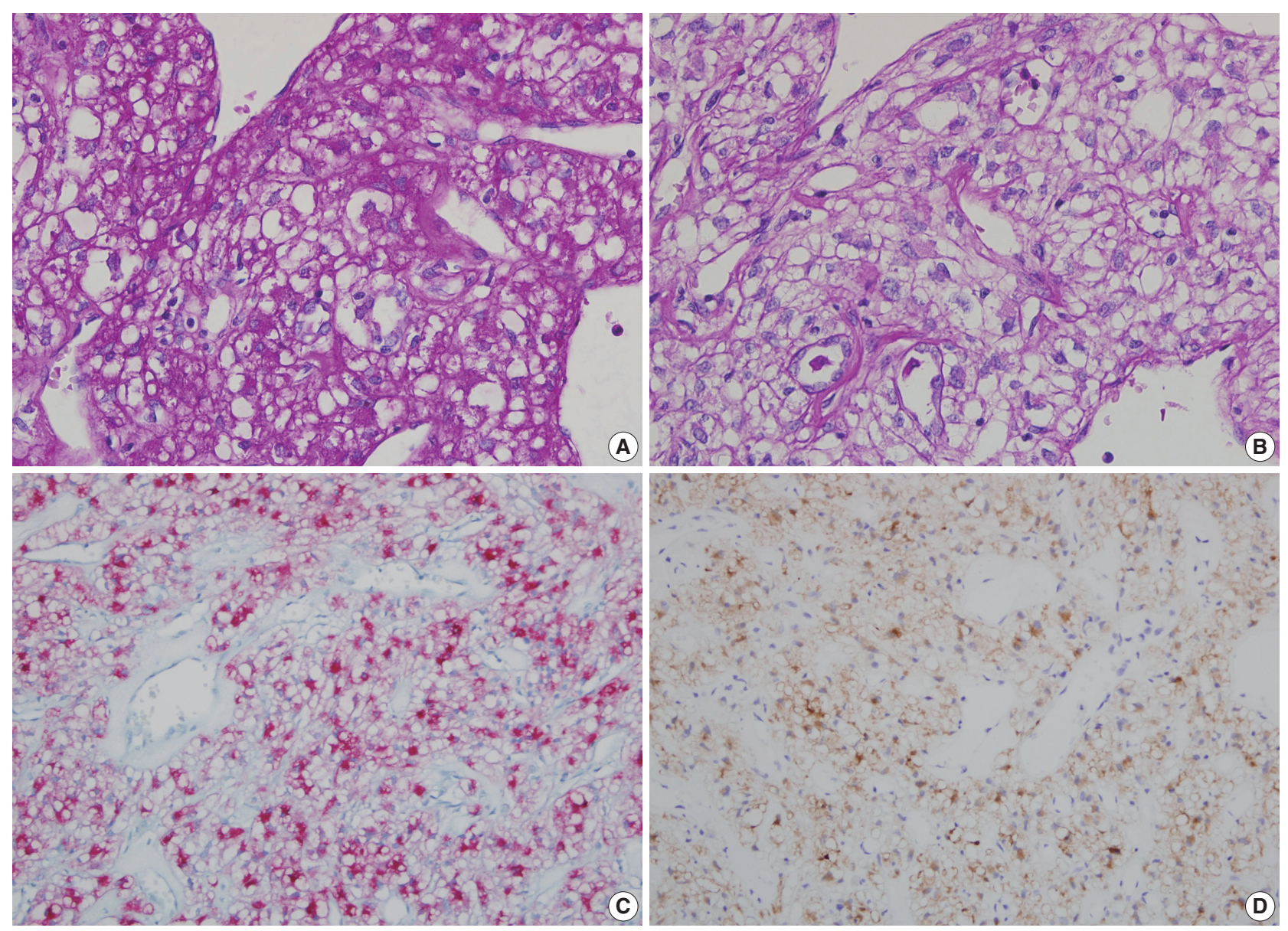

Fig. 3. Special stain and immunohistochemical stains (A, B) Periodic acid-Schiff (PAS) (A) stain and diastase-PAS (B) stain show positive reaction of the clear intracytoplasmic vacuoles in tumor cells. (C, D) Tumor cells show positive reaction to human melanoma black 45 (C) and to Melan-A (D).

reactive in mucicarmine and alcian blue stainings.

\section{Immunohistochemical findings}

Various immunohistochemical studies were performed. The results are summarized in Table 1 . Tumor cells showed positivity for HMB-45 and Melan-A (Fig. 3C, D). They were negative for S-100 protein, neuroendocrine markers (CD56, synaptophysin, and chromogranin), pulmonary adenocarcinoma markers (thyroid transcription factor 1 [TTF-1], surfactant, and napsin), and cytokeratins. Ki-67 labeling index was low $(<2 \%)$.

\section{DISCUSSION}

Benign PEComa of the lung was first described in 1963 by Leiwbow and Castleman. ${ }^{1}$ It was termed "sugar tumor" due to the intracytoplasmic glycogen content. It is a rare benign neoplasm and about 50 cases have been reported in the English literature so far.
Table 1. Results of immunohistochemical studies of the benign sclerosing perivascular epithelioid cell tumor

\begin{tabular}{|c|c|}
\hline Test & Result \\
\hline HMB-45 & Positive \\
\hline Melan-A & Positive \\
\hline S-100 & Negative \\
\hline CD56 & Negative \\
\hline Synaptophysin & Negative \\
\hline Chromogranin & Negative \\
\hline Vimentin & Positive \\
\hline TTF-1 & Negative \\
\hline Surfactant & Negative \\
\hline Napsin & Negative \\
\hline Cytokeratin & Negative \\
\hline p63 & Negative \\
\hline Ki-67 & Positivity in $<2 \%$ \\
\hline CD68 & Negative \\
\hline SMA & Positive of intratumoral vasculature \\
\hline CD34 & Positive of intratumoral vasculature \\
\hline
\end{tabular}

HMB-45, human melanoma black 45; TTF-1, thyroid transcription factor 1; SMA, smooth muscle antigen. 
Patients with benign PEComas of the lung are generally asymptomatic. ${ }^{6}$ Our case was similar to most other cases in that the patient had no symptoms and the tumor was detected incidentally. A few cases presented with hemoptysis. ${ }^{7.8}$ One case presented with thrombocytosis which resolved after the removal of the tumor. ${ }^{9}$

Typical aspirate specimens of benign PEComas show loosely cohesive clusters of bland-looking cells of various sizes. The cells are mostly epithelioid or spindle and have round-to-oval nuclei, indistinct nucleoli, and vacuolated cytoplasm. Nuclear atypia is rare and necrosis is not observed. Our findings were generally similar to the previous cytologic reports of Nguyen in $1989,{ }^{2}$ Edelweiss et al. in $2007^{4}$ and Policarpio-Nicolas et al. in 2008. ${ }^{3}$ Two exceptional findings include small distinct nucleoli and fine granular basophilic cytoplasm, while others reported indistinct or inconspicuous nucleoli and clear, vacuolated cytoplasm. Different preparation methods might have contributed to these differences. In our case, the aspiration specimen was fixed in alcohol and prepared according to the liquid-based method. Other reports on cytologic findings of benign PEComa all used conventional smear method.

Histologically, benign PEComas are composed mainly of sheets of polygonal cells and occasional spindle cells. Those polygonal cells have round to oval nuclei with granular chromatin and clear cytoplasm with distinct cell borders. The cells look bland and show low proliferative index. Multiple thin-walled and dilated vessels throughout the tumor are a characteristic feature. ${ }^{10}$ Tumor cells generally show immunoreactivity with HMB45, Melan-A, and vimentin. ${ }^{11}$ They are negative for cytokeratin, TTF-1, and neuroendocrine markers. Reactivity for S-100 protein is variable..$^{6,11,12}$

Our case was generally similar to the typical findings of benign PEComas of the lung. However, it was different from other cases in that the thin-walled vessels were surrounded by densely hyalinized stroma.

In 2008, Hornick and Fletcher ${ }^{5}$ reported an analysis on sclerosing PEComa as a distinctive variant. PEComas showing more than $50 \%$ stromal hyalinization were designated as sclerosing PEComa. Among the total 70 cases collected from 1996 to 2006, only 13 cases fulfilled the criterion. Similar to conventional PEComas, those tumors were composed of bland-looking round to oval cells with clear or eosinophilic cytoplasm. The most distinguishable feature that differed from the conventional PEComa was excessive hyalinization of the stroma. On the basis of this report, we diagnosed our case as benign sclerosing PEComa.

In the collected cases, stromal hyalinization showed cord-like, trabecular or rarely, nested growth pattern. Eight out of 13 benign sclerosing PEComas arose in retroperitoneum, while remainders were found in the abdominal wall or pelvis. ${ }^{5}$ Other reports described several cases in female genital tract and pararenal area. ${ }^{12,13}$ There has been no case depicted as benign sclerosing PEComa of the lung. In addition, no reports on cytologic findings of benign sclerosing PEComa of any organs exist to our knowledge.

When considering sclerosing variant of benign PEComa in the aspiration cytology specimen from the lung, sclerosing pneumocytoma should be included in differential diagnosis. Sclerosing pneumocytoma is usually found incidentally on chest X-ray as a single round mass. Typical cytologic findings of sclerosing pneumocytoma include blood spaces surrounded by epithelial cell-like tumor cell aggregates. Tumor cells have round to oval nuclei, finely distributed chromatin, and small nucleoli. Tumor cells may aggregate around sclerotic stromal cores. ${ }^{14}$ These findings might be confusing with those of benign sclerosing PEComa of the lung, which may show small blood vessels with surrounding neoplastic cells and hyalinizing material.

Differential diagnosis for typical benign PEComa of the lung includes primary clear cell adenocarcinoma of the lung and metastatic clear cell renal cell carcinoma. Primary clear cell adenocarcinoma of the lung shows clusters or single cells with ill-defined cell borders and pale cytoplasm. Nuclei are pleomorphic and have prominent nucleoli. Metastatic clear cell renal cell carcinoma shows round to polygonal cells which make loosely cohesive clusters. Tumor cells have abundant pale cytoplasm and round to oval nuclei. While nuclear atypia is mild in low-grade clear cell renal cell carcinoma, high-grade tumors show marked pleomorphism with occasional macronucleoli.

It is difficult to diagnose benign PEComa of the lung by fine needle aspiration only. If an asymptomatic patient has a coinshaped mass in the lung periphery, cytologic features mentioned above are helpful for the differential diagnosis of other pulmonary tumors with clear cell features. Before diagnosing sclerosing variant of benign PEComa, the possibility of sclerosing pneumocytoma should always be considered.

\section{Conflicts of Interest}

No potential conflict of interest relevant to this article was reported.

\section{REFERENCES}

1. Liebow AA, Castleman B. Benign "clear cell tumors" of the lung. 
Am J Pathol 1963; 43: 13-4.

2. Nguyen GK. Aspiration biopsy cytology of benign clear cell ("sugar") tumor of the lung. Acta Cytol 1989; 33: 511-5.

3. Policarpio-Nicolas ML, Covell J, Bregman S, Atkins K. Fine needle aspiration cytology of clear cell "sugar" tumor (PEComa) of the lung: report of a case. Diagn Cytopathol 2008; 36: 89-93.

4. Edelweiss M, Gupta N, Resetkova E. Preoperative diagnosis of clear cell "sugar" tumor of the lung by computed tomographyguided fine-needle biopsy and core-needle biopsy. Ann Diagn Pathol 2007; 11: 421-6.

5. Hornick JL, Fletcher CD. Sclerosing PEComa: clinicopathologic analysis of a distinctive variant with a predilection for the retroperitoneum. Am J Surg Pathol 2008; 32: 493-501.

6. Wang GX, Zhang D, Diao XW, Wen L. Clear cell tumor of the lung: a case report and literature review. World J Surg Oncol 2013; 11: 247.

7. Gora-Gebka M, Liberek A, Bako W, Szumera M, Korzon M, Jaskiewicz K. The "sugar" clear cell tumor of the lung-clinical presentation and diagnostic difficulties of an unusual lung tumor in youth. J Pediatr Surg 2006; 41: e27-9.

8. Santana AN, Nunes FS, Ho N, Takagaki TY. A rare cause of he- moptysis: benign sugar (clear) cell tumor of the lung. Eur J Cardiothorac Surg 2004; 25: 652-4.

9. Sen S, Senturk E, Kuman NK, Pabuscu E, Kacar F. PEComa (clear cell "sugar" tumor) of the lung: a benign tumor that presented with thrombocytosis. Ann Thorac Surg 2009; 88: 2013-5.

10. Kim WJ, Kim SR, Choe YH, et al. Clear cell "sugar" tumor of the lung: a well-enhanced mass with an early washout pattern on dynamic contrast-enhanced computed tomography. J Korean Med Sci 2008; 23: 1121-4.

11. Mizobuchi T, Masahiro N, Iwai N, Kohno H, Okada N, Nakada S. Clear cell tumor of the lung: surgical and immunohistochemical findings. Gen Thorac Cardiovasc Surg 2010; 58: 243-7.

12. Yamada $Y$, Yamamoto $H$, Ohishi $Y$, et al. Sclerosing variant of perivascular epithelioid cell tumor in the female genital organs. Pathol Int 2011; 61: 768-72.

13. Leão RR, Pereira BJ, Grenha V, Coelho H. Pararenal sclerosing PEComa. BMJ Case Rep 2013; 2013: bcr2013009097.

14. Wojcik EM, Sneige N, Lawrence DD, Ordóñez NG. Fine-needle aspiration cytology of sclerosing hemangioma of the lung: case report with immunohistochemical study. Diagn Cytopathol 1993; 9: 304-9. 